\title{
Decomposition analysis of differential dose volume histograms
}

\author{
Frank Van den Heuvel Ph.D. ${ }^{\text {a) }}$ \\ Department of Oncology and Experimental Radiation Therapy \\ Universiteit Leuven, Belgium
}

Dose volume histograms are a common tool to assess the value of a treatment plan for various forms of radiation therapy treatment. The purpose of this work is to introduce, validate, and apply a set of tools to analyze differential dose volume histograms by decomposing them into physically and clinically meaningful normal distributions. A weighted sum of the decomposed normal distributions (e.g. weighted dose) is proposed as a new measure of target dose, rather than the more unstable point dose.

The method and its theory are presented and validated using simulated distributions. Additional validation is performed by analyzing simple four field box techniques encompassing a pre-defined target, using different treatment energies inside a waterphantom.

Furthermore, two clinical situations are analyzed using this methodology to illustrate practical usefulness. A comparison of a treatment plan for a breast patient using a tangential field setup with wedges is compared to a comparable geometry using dose compensators. Finally, a normal tissue complication probability (NTCP) calculation is refined using this decomposition. The NTCP calculation is performed on a liver as organ at risk in a treatment of a mesothelioma patient with involvement of the right lung.

The comparison of the wedged breast treatment versus the compensator technique yields comparable classical dose parameters (e.g Conformity Index $\approx 1$ and equal dose at the ICRU dose point). The methodology proposed here shows a $4 \%$ difference in weighted dose outlining the difference in treatment using a single parameter instead of at least two in a classical analysis (e.g. mean dose, and maximal dose, or total dose variance). NTCP-calculations for the mesothelioma case are generated automatically and show a $3 \%$ decrease with respect to the classical calculation. The decrease is slightly dependant on the fractionation and on the $\alpha / \beta$-value utilized.

In conclusion, this method is able to distinguish clinically important differences between treatment plans using a single parameter. This methodology shows promise as an objective tool for analyzing NTCP and doses in larger studies, as the only information needed is the dose volume histogram.

\section{INTRODUCTION}

In the planning of external radiation therapy treatment the use of cumulative dose volume histograms (cDVH) are a much used resource ${ }^{1}$. In the subjective process of determining the best treatment plan a physician can use the cDVH's to: 1) judge adequate tumor coverage, 2) choose the optimal isodose line for prescription, 3) assess the volume of high doses in critical organs, 4) calculate normal tissue complication probabilities in heterogeneously irradiated organs ${ }^{2}$, and 5) optimize treatment plans in inverse treatment planning, using a crude representation of cDVH's ${ }^{3-5}$.

This paper introduces a more refined model, based on normal distributions, of the differential dose volume histograms (dDVH), and by extension cDVH's. This model also allows a more quantitative approach to the use of DVH's by defining the dose (as given by the parameters of the distribution: mode, width and scale) delivered to a target in a more rigorous fashion.

Using this definition it becomes possible to

- Renormalize dose data from different planning methodologies thereby diminishing the role of the normalization point.

\footnotetext{
a)Electronic mail: frank.vandenheuvel@uz.kuleuven.ac.be
}

- Quantify the inhomogeneity of a dose distribution.

- Robustly quantify the NTCP of an irradiated organ, and refine the calculation to include differences in fractionation over an inhomogeneously irradiated organ

The currently available methods of dose prescription and reporting all have considerable limitations. One widely used parameter is the Conformity Index (CI ${ }^{6}$. The CI is defined as the ratio of the volume of the target receiving the prescription dose to the total volume of the target $C I=V_{T, P} / V_{T}$. It is clear that this index well describes the fact that a target receives enough dose. However, it does not provide any information regarding doses higher than the prescription dose. Other indicators like minimal and maximal dose $\left(d_{\min }, d_{\max }\right)$ are point based and subject to variations by choice of normalization and choice of points as well as grid size. The mean dose $\left(d_{\text {mean }}\right)$ is only a good indicator when the dose distribution is uniform (e.g. a normal distribution). It fails to provide adequate normalization in cases where doses the target volume are highly variable.

In this paper we provide a physical background for the decomposition, by applying it to a simulation and to simple treatment plan with increasing degree of inhomogeneity. Furthermore, two clinical applications are presented, demonstrating the possible use in comparing plans with different homogeneity in breast treatment and providing a refined methodology to calculate normal tissue compli- 
cations for the liver as an organ at risk in treatment of mesothelioma.

\section{THEORY}

We start out from the observation that the dose in a plane perpendicular to a beam and limited to about 1 $\mathrm{cm}$ from the field edges, behaves as a normal distribution around a given mean. The spread of this normal distribution depends on the beam flatness and the inherent variation of the beam measurement, which again is a normal distribution. In practice, a target volume is treated by combining such beams to obtain a homogeneous dose distribution in that volume. The resulting dose distribution can be approximated by a mean dose with a small deviation characterized by a normal distribution. The determination of a volume dose histogram is done by sampling the volume of interest using a random seed method or, alternatively, a grid--method ${ }^{7,8}$, and determine the volume that a given dose level encompasses.

The previous paragraph suggests that the differential dose volume histogram of a homogeneously irradiated organ (e.g. a constant dose with some normal variation ), or an organ part, can be modeled as a normal distribution. Here the expression of the frequency distribution or Gauss-curve is used:

$$
\phi(x)=\frac{1}{\sqrt{2 \pi} \sigma} e^{-\frac{1}{2}\left(\frac{x-x_{0}}{\sigma}\right)^{2}}
$$

Where $\sigma$ is the standard deviation and $x_{0}$ is the mode (in the case of a normal distribution, the mode is mathematically equivalent to the mean as well as the median of the distribution). The interpretation of the two parameters is straightforward: $\sigma$ is an estimate of the homogeneity of the dose delivered to the structure and $x_{0}$ is the dose delivered to the majority of the points within the structure and hence represents an estimate of the dose delivered to that volume.

The expression as given in equation 1 is normalized to a volume of unity in the differential dose volume histogram, which is equivalent to having unity area under the curve in a dDVH. A multiplicative scale factor $S$ provides a variable to take into account the volume of the irradiated structure.

Additionally, we hypothesize that a dose distribution in any irradiated organ is made up of a finite number of homogeneous dose regions that can be described by the expression in equation 1. A dDVH of such an organ can therefore be modeled as a superposition of Gauss-curves. Note that splitting up DVH's in homogeneous parts is not new. Kutcher et $\mathrm{al}^{9}$ introduced this to provide normal tissue complication factors for inhomogeneous irradiated organs, which is outlined in the following section:

\section{A. Normal Tissue Complication of non-uniformly irradiated organs.}

We start from the expression of NTCP as proposed by Lyman et al.:

$$
N T C P=\frac{1}{2 \pi} \int_{-\infty}^{t} \exp \left(-\frac{x}{2}\right) d x
$$

where

$$
t=\frac{D-T D_{50}(v)}{m T D_{50}(v)}
$$

In equation $3, D$ denotes the total dose delivered in 2 Gy fractions to the volume under consideration. The parameter $m$ determines the steepness of the dose-response curve. The factor $T D_{50}(v)$ is the dose level (in $2 \mathrm{~Gy}$ fractions) where $50 \%$ of the population exhibits complications. The parameter $v$ is introduced to allow partial irradiation of organs. A value $v=1$ implies complete irradiation of the organ at a given level. Any $v<1$ implies partial irradiation of that fraction of the organ to the given level, the remainder of the organ receives no dose. As not every organ has the same response to partial irradiation another parameter $n$ is introduced like so:

$$
T D_{50}(v)=T D_{50}(1) v^{-n}
$$

The previous NTCP-calculation method is only for homogeneous or partial but homogeneously irradiated volumes. The effective volume method divides the organ in volumes of homogeneous dose and adds them using a weighted sum as follows:

$$
V_{e f f}=\sum_{i} V_{i}\left(\frac{D_{i}}{D_{\max }}\right)^{\frac{1}{n}}
$$

With $V_{i}$ the various sub-volumes homogeneously irradiated with dose $D_{i}, D_{\max }$ the maximal dose in the organ and $n$ a volume-effect parameter. The parameter $v$ or the fraction of the volume irradiated now becomes:

$$
v=\frac{V_{e f f}}{\sum_{i} V_{i}}
$$

Note that Equation 5 depends directly on the value of $D_{\max }$, which could generate different results for DVH's that are very similar. Furthermore, this model does not take into account the fact that each subvolume receives a dose in a different fractionation schedule. If, for example, an organ receives a dose of 50 Gy in 2 Gy fractions, then the sub-volume that receives half the dose, will receive 25 Gy in fractions of 1 Gy. The method proposed by Kutcher et al. does not use a well-defined concept of a homogeneous dose. It is left to the implementer to decide which parts of the dose volume histogram constitute a homogeneous region. 


\section{B. BED - rescaling}

Decomposition analysis of dDVH's allows to implement $\mathrm{NTCP}-$ calculations in a more rigorous fashion. The result of the decomposition can be written as a series of total doses $\left(D_{i}\right)$, each linked to a volume $\left(V_{i}\right)$. If in a given decomposition we obtain $N$ such peaks, then we obtain two series $\left(D_{1}, D_{2}, \ldots, D_{N}\right)$ and $\left(V_{1}, V_{2}, \ldots\right.$, $\left.V_{N}\right)$ ordered from low to high dose. When calculating NTCP we need to convert each dose to a total dose delivered in 2 Gy fractions. This can be achieved using the Biological Effective Dose (BED) methodology, using the well known expression from the linear quadratic model:

$$
B E D=D\left(1+\frac{d}{\alpha / \beta}\right)
$$

Where $D$ is the total dose, $d$ the dose per fraction, and $\alpha$ and $\beta$ the tissue specific radiobiological response factors ${ }^{10}$.

Applying equation 7 to the dose series yields a new series: $\left(B E D_{1}, B E D_{2}, \ldots, B E D_{N}\right)$ which can in turn, using the same expression, be transformed to $\left(D_{1}^{\prime}, D_{2}^{\prime}\right.$, $\ldots, D_{N}^{\prime}$ ), with $D_{i}^{\prime}$ being the total dose in 2 Gy fractions delivered to partial volume $V_{i}$. These values are used as dose to calculate the effective volume as in equation 5 :

$$
V_{e f f}=\sum_{i=1}^{N} V_{i}\left(\frac{D_{i}^{\prime}}{D_{N}^{\prime}}\right)^{\frac{1}{n}}
$$

Once the $V_{\text {eff }}$ is obtained, the classical NTCPcalculation as described above is carried out.

\section{Renormalization}

BED-renormalization uses the BED expression (Equation 7 ) to calculate the $D_{\max }$ dose to a total dose delivered in fractions of $2 \mathrm{~Gy}$. The effective volume is calculated as before (the ratio is assumed not to change).

\section{IMPLEMENTATION}

The implementation of the fit takes place in two steps.

\section{A. Fit procedure}

A slightly altered form of equation 1 is used to perform the fit:

$$
O(x)=X_{1} e^{-\left(\frac{x-X_{2}}{X_{3}}\right)^{2}}
$$

Where

$$
\begin{aligned}
& X_{2}=x_{0} \\
& X_{3}=\sqrt{2} \sigma
\end{aligned}
$$

and

$$
X_{1}=\frac{S}{\sqrt{2 \pi} \sigma}
$$

The term $X_{1}$ now denotes the maximal height of the gauss curve. The scale factor $S$ denotes the area under the curve and thus estimates the volume of the irradiated structure.

1. The dDVH is populated with normal distributions with a standard deviation of $1 \%$ of the prescribed dose. The different distributions are spaced $1.33 \%$ apart and are normalized to unity. The different contributing Gauss-curves are identified by performing a fit of expression 9 in a limited dose range (twice the dose spread). The Gauss-curve is optimized using the three parameters $\left(X_{1}, X_{3}, X_{3}\right)$.

2. Re-optimization of all parameters of the sum of all Gauss-curves starting from the values found in the previous step, is performed. No dose-range restrictions are imposed in this step. All parameters are restricted to positive values. Peaks representing volumes smaller than $0.001 \%$ of the total volume are discarded as they are comparable to the roundoff errors in the computer system.

In this implementation all fits are performed using an implementation of the nonlinear least-squares MarquardtLevenberg algorithm as implemented in the open source gnuplot ${ }^{\text {? }}$ software $^{11}$. We propose a straightforward weighted combination of peaks to describe a dose distribution with multiple peaks. Let $\left(x_{0}\right)_{i}, \sigma_{i}$ and $S_{i}$ be, respectively, the mode, width, and scale of the i-th curve, then:

$$
\left(x_{0}\right)_{t o t}=\sum_{i=1}^{N} \frac{\left(x_{0}\right)_{i} S_{i}}{\sum_{i=1}^{N} S_{i}}
$$

And following the same rational

$$
\sigma_{t o t}=\sum_{i=1}^{N} \frac{\sigma_{i} S_{i}}{\sum_{i=1}^{N} S_{i}}
$$

In this particular implementation it is possible that some initiated curves do not contribute to the overall shape. The number of contributing Gauss-curves is not a constant, as curves with contributions smaller than $0.001 \%$ are discarded.

\section{B. Generation of cumulative dose volume histograms}

Once the different peaks have been found, a cumulative version of the DVH can be generated using the standard expression for an error function $\operatorname{erf}(x)=\frac{2}{\pi} \int_{0}^{x} \exp ^{-\left(t-x_{0}\right)^{2}} d t$. scaled with the correct scaling value. To obtain the form we are used to, we need to plot $1-\operatorname{erf}(x)$. 


\section{METHODS AND MATERIALS}

\section{A. Validation}

\section{Simulation}

In order to show that our fitting software works adequately we perform two simulations using generated data sets.

A dose distribution with normal variation is generated using a hit-and-miss Monte Carlo method. The mean of the distribution $\left(x_{0}\right)$ is set at 74 Gy and the standard deviation $(\sigma)$ is chosen to be 1 Gy. A differential dose volume histogram is generated from this data set. A Gauss-curve is fit to the dDVH. For a second simulation three normal distributions are sampled randomly generating a more complicated $\mathrm{dDVH}$. The distributions have respective means of $73.0,74.0$, and 75.0 with standard deviations of respectively: $1.0,3.5$, and 0.5 , with arbitrary units.

As a measure of the goodness of fit we use the reduced $\chi^{2}$-value, defined as the sum of the square of the residuals normalized to the degrees of freedom of the fit. For an acceptable fit this number should be close to one or smaller. We further denote this value as "RMS".

\section{Phantom Study}

To validate the assumptions, and illustrate the usefulness of the quantification we generated the following treatment plan.

A water phantom is simulated in a radiation planning system. A classic four field box using a 10x10 $\mathrm{cm}^{2}$ square field is generated to irradiate a $50 \times 50 \times 50 \mathrm{~cm}^{3}$ water phantom. We define a target inside the box allowing for a $1 \mathrm{~cm}$ penumbra and define the normalization point as the centroid of the target. The point was normalized to $100 \mathrm{cGy}$. The procedure is repeated for three different nominal energies: 6, 10, and $15 \mathrm{MV}$. We know from experience and inspection that the use of these respective energies will yield plans with increasing homogeneity.

A dDVH is generated from the data in the previous paragraph after which equation 1 or a linear combination thereof is fit to the data.

\section{B. Patient Studies}

In this section we apply Gauss-peak analysis to the dDVH's of clinical data to illustrate possible uses.

1. A comparison is made of different plans for a breast patient using wedges or compensators.

2. An assessment of normal tissue complication probability for a mesothelioma treatment of the right lung involving substantial dose in the liver, is performed.

\section{Breast Treatment}

For illustrative purposes a comparison is made between two treatment approaches that are known to have different levels of homogeneity in the target volume ${ }^{12-14}$. A patient with carcinoma of the breast is planned for treatment with opposing tangential beams. Half beam blocking is used to align the beams. The angle of the beam is chosen so as to minimize the amount of irradiated lung tissue. A normalization point is chosen at 1.5 $\mathrm{cm}$ from the central axis. The beams are weighted by the dosimetrist to get the best distribution in the isodose display. For the wedged plan, the dosimetrist uses trial and error combinations of a set of fixed wedge angles in both beams. The possible angles are: $15^{\circ}, 30^{\circ}, 45^{\circ}$ and $60^{\circ}$. The compensator is calculated to provide a uniform dose at the midplane perpendicular to the beam axis (i.e. a dose compensator rather than a missing tissue compensator). Subsequently, the physician reviews the plan and based on the isodose presentation, the cDVH for the target, and the cDVH's for the critical structures (e.g. the lung) selects the appropriate dose level. The plans are normalized to the same point 1.5 from the central axis. The dDVH analyzed here is normalized to $100 \%$ at the normalization point.

As the plans generated here are from a clinical setup, there was no "target" volume present in the treatment plan. The target volume was added after the plan was finished. For the target and the ipsilateral lung a dDVH was generated with a resolution of $0.1 \%$. The $\mathrm{dDVH}$ is generated by seeding 10,000 random points within the volume.

\section{Lung Patient}

Patients treated for mesothelioma of the right lung usually receive a substantial dose to the liver. Kutcher et.al developed a methodology to calculate the normal tissue complication factors ${ }^{2}$. This was applied by McGinn et al. to define the dose for treatment of interhepatic cancers allowing an NTCP of $10 \%^{15}$. Although mathematically, this approach is sound and straightforward, it does not take into account the fact that fractionation will be different at each point in the DVH. Using the methodology developed above it now becomes possible to modify the dDVH to reflect the impact of fractionation by converting all of the doses to biological effect dose (BED) and back to total dose delivered with fractions of 2 Gy. Now that we have identified volumes of homogeneous dose we can include the fractionation differences in the $\mathrm{dDVH}$ for every volume separately by converting them to BED. Furthermore, it becomes possible to generate different DVH's for different fractionation schemes. This subsection provides an example of this approach in a clinically relevant case.

A patient was treated for mesothelioma of the right lung using 1.8 Gy fractions to a total dose of 50.4 Gy. A 
dDVH was calculated for the dose delivered to the liver. A Gauss-peak analysis was performed using 53 curves. Every curve was adjusted to reflect the correct BED depending on it's contribution to that fraction using equation 7 . The $\alpha / \beta$-ratio was considered as a parameter and a number of "interesting" values were used. The original data was normalized to reflect the correct total dose in the treated fractionation. The maximal dose as required in equation 5 is given by the mode of the Gaussian decomposition with the highest dose.

Not only does the $\alpha / \beta$-ratio change the NTCP, it also changes the impact of the fractionation schedule. This relationship is investigated by plotting the NTCP as a function of the $\alpha / \beta$-ratio for different fractionation schemes.

\section{RESULTS}

\section{A. Simulation}

Both the single distribution as well as the combination of several normal distributions are modeled correctly by the software, as shown in Tables I and II. Figure 1 shows the fit and decomposition. The RMS values for both simulations are adequately low.

\section{B. Phantom study}

Figure 2 shows the resulting dDVH's with the various Gauss-curves that contribute to the overall shape. Full results are tabulated in table III. The $15 \mathrm{MV}$ plan is approximated for $93 \%$ by a large Gauss-curve and two additional smaller scaled curves contributing respectively $5 \%$ and $2 \%$ of its volume. The $10 \mathrm{MV}$ plan necessitates larger contributions from the extra peaks. The $6 \mathrm{MV}$ plan needs an additional peak to describe its dDVH.

Also note that although all plans were normalized to an identical geometric point, the weighted dose sum as defined in Formula (13) provides differences of up to $2 \%$. Renormalization to equalize the weigthed dose sum in the $10 \mathrm{MV}$ and $15 \mathrm{MV}$ plans yields almost identical dDVH's (not shown).

\section{Patient Study}

\section{Breast treatment plan}

The use of Gauss-peak analysis for a target structure is straightforward. The mode of the distribution $\left(x_{0}\right)$ represents the dose delivered to the majority of the considered volume. This makes it a prime candidate as a quantifier for the dose delivered to the structure. In this case the dose to the target structure is $101.9 \%$ for the compensator case and $104.2 \%$ for the wedged treatment. This while using exactly the same normalization point.
Note that the plan was normalized to $100 \%$ in a single point, the subsequent analysis will then be done on a $\%$ scale. The complete decomposition is listed in Table IV.

The width of the contributing Gauss-curves was not limited in this particular implementation . Therefore a number of Gauss-peaks were replaced by two larger peaks.

The analysis of critical structures shows a more complex dDVH (Fig 3). In this case we can identify two separate peaks (i.e. a set of overlapping Gauss-curves). We define the peak with the highest weighted mode as the hotspot. The area under the selected peak then gives the volume of the hot spot.

\section{BED corrected NTCP}

Figure 4 shows the effect of BED-corrected dDVH's. Normal tissue complication factors were then calculated as suggested by McGinn et al. ${ }^{15}$. Table V shows the impact of the correction and the fractionation on the NTCP. In this table we see that the corrected NTCP is systematically smaller than the uncorrected. The level of decrease depends on the $\alpha / \beta$-value. Figure 4 shows the influence of this factor on the various fractionation schemes. This graph clearly shows that in hypo-fractionated regimes small changes in $\alpha / \beta$ values change the NTCP values substantially.

\section{DISCUSSION}

We have shown that it is possible to perform a more refined analysis of a dDVH (cDVH) of a treatment plan and draw clinically significant conclusions.

In the simulation part the algorithm was shown to be able to adequately distinguish a number of distributions in a combined treatment.

The phantom study showed that such an analysis can identify subtle differences in treatments that are very close in terms of homogeneity. These differences have clinical impact when point based normalizations are used. Differences of up to $2 \%$ in delivered dose were identified. Renormalization using the above methodology shows nearly identical dDVH's. Currently, most commercially available planning systems still insist on point based normalization and use reference points to represent a treatment? . Monte Carlo based systems on the other hand are not yet widespread. In the latter systems it is not possible to define the dose at a point as there is no physical basis for this, hence the difficulty in providing monitor units in such systems.

The fact that the $15 \mathrm{MV}$ four field box results are well described by a single normal distribution function, corroborates our hypothesis. As mentioned in the theory section we observed that a dose distribution in a plane can be described by a normal distribution. The cause for this distribution is the flatness of the beam, variations in 


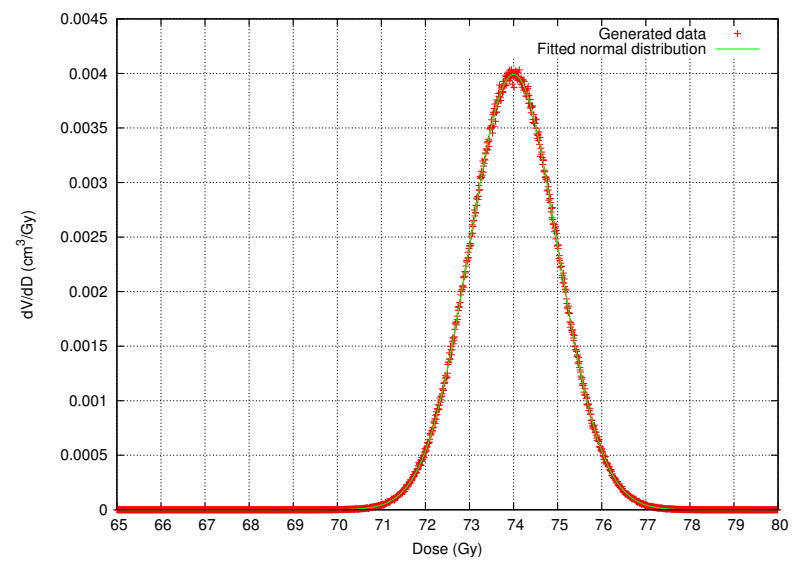

(a)single peak at $74 \mathrm{~Gy}$

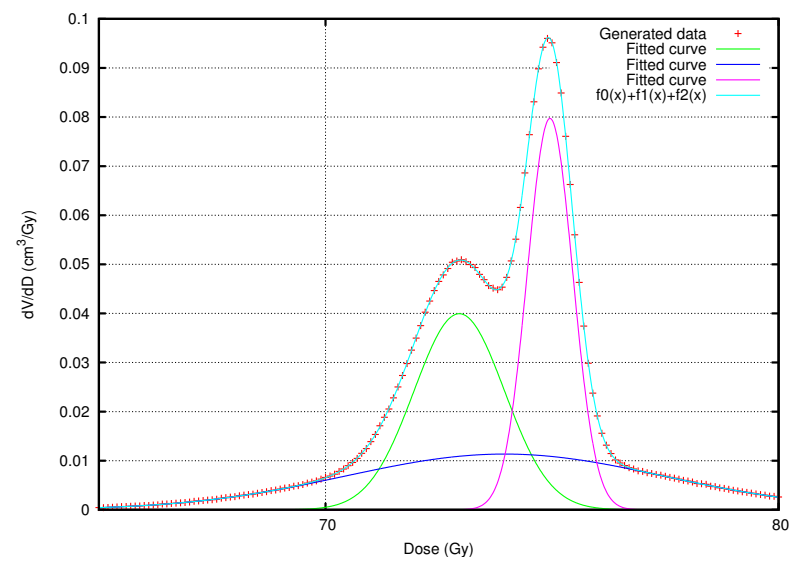

(b)Combination of 3 distributions

FIG. 1. Differential dose volume histogram of a $5 \times 5 \times 5 \mathrm{~cm}^{3}$ box with simulated dose distributions to test the fit program

\begin{tabular}{|c|c|c|c|}
\hline & Simulated values distribution & Fit parameters & Fit error \\
\hline Mean: & 74.00 & 74.0006 & 0.0005 \\
\hline$\sigma$ & 1.00 & 0.99941 & 0.0005 \\
\hline Scale & 25.00 & 25.066 & $1.68310^{-6}$ \\
\hline
\end{tabular}

TABLE I. A dose distribution was generated in a box with a volume of $25 \mathrm{~cm}^{3}$ following a normal distribution with mode 74 Gy and standard deviation $(\sigma)$ of 1 Gy. The second column shows the parameters of the performed fit.

Simulated values distribution Fit parameters Fit error

\begin{tabular}{l|ccc}
\hline \hline Mean & 73.0 & 72.953 & 0.001 \\
$\sigma$ & 1.0 & 0.999 & 0.002 \\
Mean & 74.0 & 73.950 & 0.007 \\
$\sigma$ & 3.5 & 3.505 & 0.010 \\
Mean & 75.0 & 74.9553 & 0.0004 \\
$\sigma$ & 0.5 & 0.5020 & 0.0004 \\
\hline \multicolumn{4}{l}{} \\
\hline
\end{tabular}

TABLE II. Simulation as in Table I with three superimposed Gauss-curves. The program correctly identifies all curves

\begin{tabular}{ccc|ccc|ccc}
\multicolumn{3}{c|}{$6 \mathrm{MV}$} & \multicolumn{3}{c|}{$10 \mathrm{MV}$} & \multicolumn{3}{c}{$15 \mathrm{MV}$} \\
\hline \hline$x_{0}(\mathrm{cGy})$ & $\sigma(\mathrm{cGy})$ & Volume & $x_{0}(\mathrm{cGy})$ & $\sigma(\mathrm{cGy})$ & Volume & $x_{0}(\mathrm{cGy})$ & $\sigma(c G y)$ & Volume \\
\hline 98.38 & 0.88 & 0.17 & 98.31 & 0.56 & 0.127 & 101.49 & 0.14 & 0.052 \\
99.61 & 0.52 & 0.20 & 99.36 & 0.33 & 0.699 & 100.14 & 0.40 & 0.929 \\
100.89 & 0.47 & 0.45 & 99.89 & 0.83 & 0.177 & 101.71 & 0.77 & 0.022 \\
102.01 & 0.54 & 0.19 & & & & & & \\
\hline $\mathbf{1 0 1 . 4 3}$ & $\mathbf{0 . 5 7}$ & $\mathbf{1 . 0 0 2}$ & $\mathbf{9 9 . 6 1}$ & $\mathbf{0 . 4 5}$ & $\mathbf{1 . 0 0 2}$ & $\mathbf{1 0 0 . 5 5}$ & $\mathbf{0 . 4 0}$ & $\mathbf{1 . 0 0 3}$ \\
\hline \hline
\end{tabular}

TABLE III. Results for homogeneous dose distributions delivered with a four-field box geometry. The results in bold are calculated using the weighted sum definitions as outlined in Equations 13 and 14 


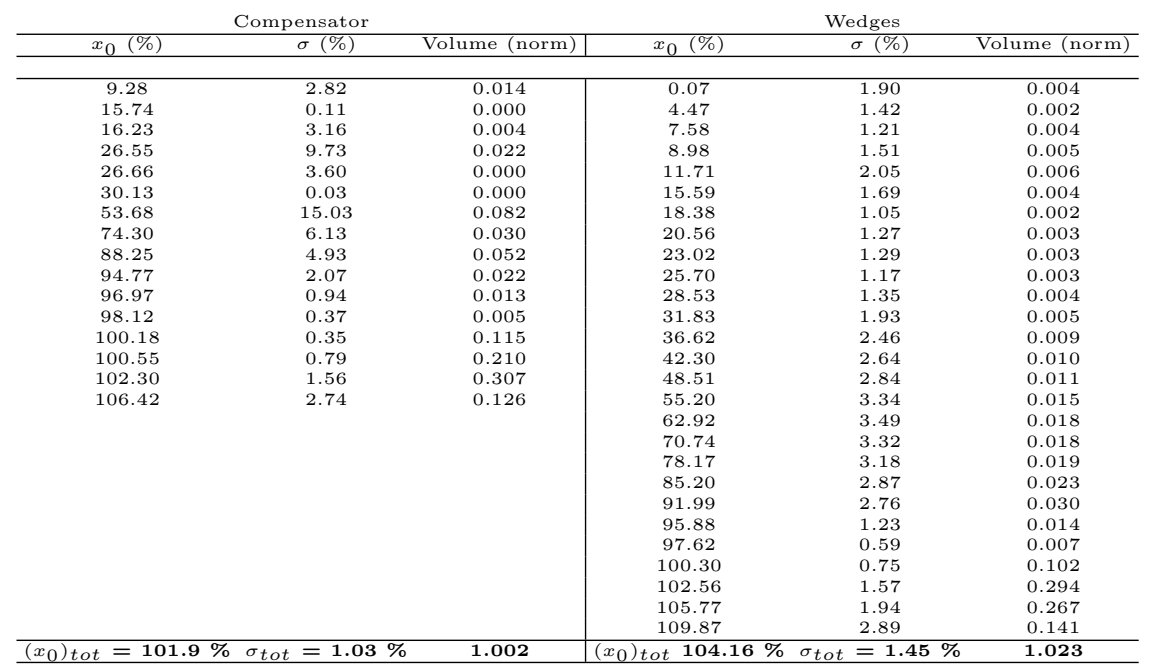

TABLE IV. Gauss-peak analysis of the dDVH of the target for a breast treatment. Both treatments show slight underdosage due to the a posteriori definition of the target volume. Homogeneity of the dose distribution can be assessed by looking at $\sigma_{\text {tot }}$-value. contributions. In this case the dDVH was a normalized one. The normalized dose was $100 \mathrm{~Gy}$, making exchange of $\%$ and Gy possible. In the compensator plan two broader peaks (with small contributions) replace a number of peaks. This artifact due to optimization can be avoided by limiting the variability of the $\sigma$ parameter in the optimization routine.

\begin{tabular}{l|ccc} 
& \# of fractions & $\alpha / \beta$ & NTCP \\
\hline \hline BED Normalized & 28 & 3 & 0.070 \\
BED Normalized & 28 & 10 & 0.070 \\
BED Corrected & 28 & 3 & 0.032 \\
BED Corrected & 28 & 10 & 0.052 \\
\hline BED Normalized & 56 & 3 & 0.006 \\
BED Normalized & 56 & 10 & 0.028 \\
BED Corrected & 56 & 3 & 0.003 \\
BED Corrected & 56 & 10 & 0.023 \\
\hline BED Normalized & 14 & 3 & 0.729 \\
BED Normalized & 14 & 10 & 0.272 \\
BED Corrected & 14 & 3 & 0.444 \\
BED Corrected & 14 & 10 & 0.177
\end{tabular}

TABLE V. Table of NTCP values with a BED corrected dDVH. Additionally, two alternative fractionation schedules are shown. Note that for all fractionations the corrected NTCP is systematically lower. The maximal value serving as the reference dose was the highest peak found (56.20Gy, which was delivered in 2 Gy fractions). The other fractionations were recalculated to reflect 2 Gy fractions also using the linear quadratic model with the tabulated $\alpha / \beta$ ratio.

the dose measurement and variations in the beam during the measurement. The first item can be considered as structured noise. By modeling the beam in a treatment planning system all these variations become static, but are still well described by the expression for a normal distribution. The hypothesis for this paper then becomes:

A volume is never treated to a single dose but rather to a range of doses, that can be described with a normal distribution function, which in turn can be estimated from the differential dose volume histogram.

It is interesting to note that much of the data pro- vided from cell-cultures to confirm the viability of radiobiological models is gleaned from experiments were cells are irradiated or alternatively from clinical data from "homogeneous" radiation treatments. The doses and fractionations reported by these researchers thus come from experiments with comparable distributions as the ones defined above.

In what way does the analysis presented here improve the classical approaches in the case of dose analysis of the target? It is clear that the spread parameter $\left(\right.$ sigma $_{\text {tot }}$ does not add a great deal when for instance range of dose or the variance of the dose is known. The only advantage one can think of is the fact that it can be determined from the dDVH rather than asked from the treatment planning system. It is therefore a parameter that could be useful if data obtained in a study is limited.

The weighted dose as defined in equation 13 has some advantages with respect to the classical approaches in that it unifies a number of parameters. For example to compare the target coverage in the breast example one needs information on the dose delivered as well as the range of the treatment. Whenever more than a single parameter is used to evaluate a system an adequate expression needs to be found combining both parameters. In the treatment proposed here only a single parameter (the weighted dose) is needed as shown below.

The two approaches for treatment of breast carcinoma both have a Conformity Index close to 1 . It is clear, however, that both treatments are not equivalent. To quantify this we have to evaluate another parameter, for example, a inhomogeneity index or a maximal dose. Adding more parameters to an interpretation problem is not conducive for objective analysis. When using the proposed methodology a single parameter is sufficient to distinguish both approaches. 


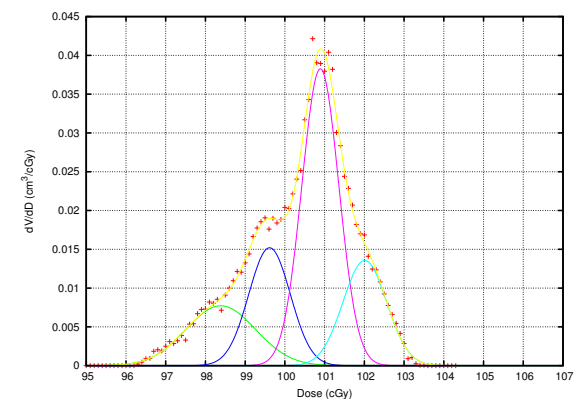

(a) $6 \mathrm{MV}$

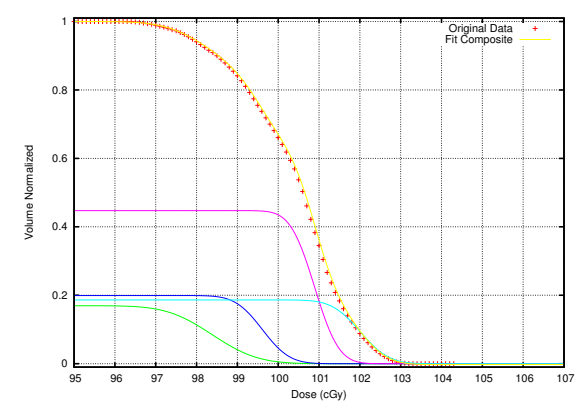

(d) $6 \mathrm{MV}$ cumulative

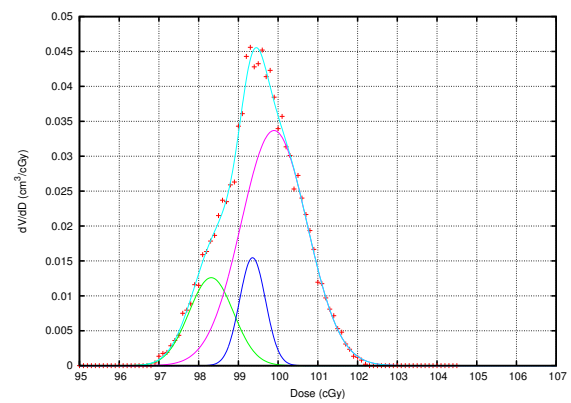

(b) $10 \mathrm{MV}$

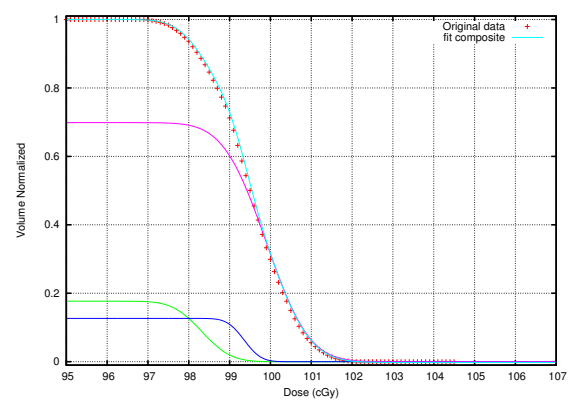

(e) $10 \mathrm{MV}$ cumulative

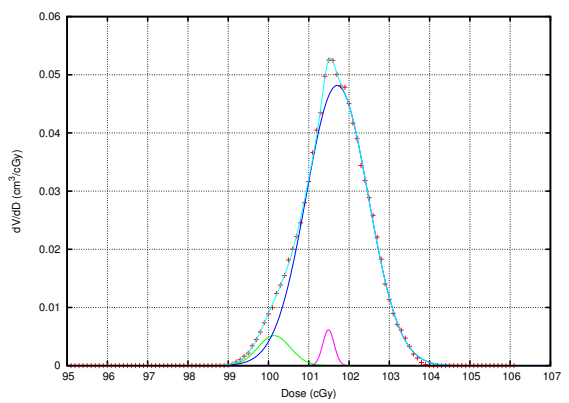

(c) $15 \mathrm{MV}$

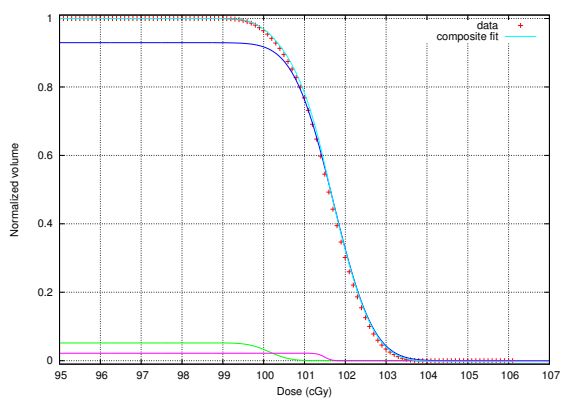

(f) $15 \mathrm{MV}$ cumulative

FIG. 2. In these graphs the same target volume was treated using a four field box with respectively 6,10 and $15 \mathrm{MV}$ photon beams. The dots are the original values from the planned treatment. The solid curve through the points is the fitted curve, while the smaller components are also shown. It is clear that the inhomogeneity for the $6 \mathrm{MV}$ beam is higher than the 10 and $15 \mathrm{MV}$ treatments. Noteworthy is that the most likely dose to the structures is significantly different (100.82, 99.56, and $101.66 \mathrm{cGy}$ ) for the different energies. This despite the fact that identical geometries and normalization points were used. The cumulative curves were generated by adding the error function expressions normalized for the various parameters, with the error function defined as $\operatorname{erf}(x)=\frac{2}{\pi} \int_{0}^{x} \exp ^{-\left(t-x_{0}\right)^{2}} d t$ 
In the future, it might be possible to extend the NTCP-calculation approach proposed here to target volumes. This would allow a unified analysis of treatments with varying inhomogeneity. As an example we can think of brachytherapy, intensity modulated treatments and classical 3D-conformal treatments, but also linacbased stereotactic treatments can be compared with gammaknife treatments.

In the analysis of the compensator plan, two broad peaks, albeit with small contributions, stand out (see Table IV). The existence of these peaks is a consequence of not limiting the spread of contributing Gausscurves. The optimization program therefore replaced many smaller peaks with one larger peak if the difference is negligible in the overall optimization.

It is clear that the optimization (e.g. selection of modes, scales and spreads) could be more refined. The fit methodology used here is fairly straightforward and could be subject to problems like converging to local minima, given the numbers of parameters that can be fit. In the future we expect that more sophisticated stochastic methods will be applied, like: simulated annealing or genetic algorithms. This is the more apparent in the analysis of the wedge plan where the total volume of the all the decomposed peaks is slightly higher than the expected normalized volume, by about $2.3 \%$.

The shape of the dDVH of the liver in the mesothelioma case is much more complicated than any of the other cases shown here. Still, an adequate fit is found. In this case the fit took more computing time than the other cases ( $\simeq 25$ hours on a AMD64, $1.8 \mathrm{GHz}$ processor, running a native 64 bit Linux kernel). This amount of time would be detrimental if many cases were to be analyzed. However, using values of a successful fit on a comparable treatment as initial values yielded results in a matter of minutes. This fact makes this approach usable to analyze data cohorts (for instance: all liver data for right lung mesothelioma) as most of these treatments will have roughly the same shapes in $\mathrm{dDVH}$.

The relationship between the $\alpha / \beta$-ratio and the fractionation scheme in the case of liver complications is not a straightforward one. From Figure $4 \mathrm{~b}$ it is clear that $\alpha / \beta$ has almost no impact on the NTCP as the dose per fraction stays low or at the 2 Gy level. When larger fractions are used $\alpha / \beta$ becomes a more important factor, especially when small values are used. It is therefore important to keep the variability of our knowledge of $\alpha / \beta$-ratios in mind when experimenting with hypofractionated regimens.

Up until recently there was a consensus that dose effect was essentially linear, in the sense that it could be added and that small doses had small effects. However, there are indications that there are different biological effects when irradiating with very small fractions ${ }^{16}$. Using a decomposition like this can bring these effects into account. A hypothetical example would be an increased risk for inducing secondary tumors for a very low dose per fraction. This could be planned in a dose volume histogram analysis by weighting the Gauss-curves differently for lower dose contributions when calculating that risk. This means that we can now apply a lookup table of risk to a treatment and that lookup table does not have to be linear.

\section{ACKNOWLEDGMENTS}

Acknowledgments should go to Adam Tazi, a student at the Wayne state university medical physics program, for being inquisitive. Professors Yolande Lievens and Karin Haustermans of the Catholic University at Leuven, Belgium are acknowledged for providing patient data for mesothelioma patients. Ms Harriet Jaenisch of the Barbara Ann Karmanos Cancer Institute for the breast planning. Professor Michael Joiner at the Barbara Ann Karmanos Cancer Institute is acknowledged for enlightening discussions. I would also like to acknowledge the reviewers and editors of Medical Physics, for their very constructive approach to this work.

${ }^{1}$ R. E. Drzymala, R. Mohan, L. Brewster, J. Chu, M. Goitein, W. Harms, and M. Urie. Dose-volume histograms. Int. J. Radiat. Oncol. Biol. Phys., 21:71-78, May 1991.

${ }^{2}$ G. J. Kutcher and C. Burman. Calculation of complication probability factors for non-uniform normal tissue irradiation: the effective volume method. Int J Radiat Oncol Biol Phys, 16:16231630, June 1989.

${ }^{3} \mathrm{M}$. Langer and J. Leong. Optimization of beam weights under dose-volume restrictions. Int. J. Radiat. Oncol. Biol. Phys., 13:1255-1260, August 1987.

${ }^{4}$ J. O. Deasy. Multiple local minima in radiotherapy optimization problems with dose-volume constraints. Medical Physics, 24:1157-1161, July 1997.

${ }^{5}$ Qiuwen $\mathrm{Wu}$ and Radhe Mohan. Algorithms and functionality of an intensity modulated radiotherapy optimization system. Medical Physics, 27(4):701-711, 2000.

${ }^{6}$ Tommy Knoos, Ingrid Kristensen, and Per Nilsson. Volumetric and dosimetric evaluation of radiation treatment plans: radiation conformity index. Int. J. Radiat. Oncol. Biol. Phys., 42:11691176, December 1998.

${ }^{7}$ Andrzej Niemierko and Michael Goitein. Random sampling for evaluating treatment plans. Medical Physics, 17(5):753-762, 1990.

${ }^{8} \mathrm{X}-\mathrm{Q}$. Lu and L. M. Chin. Sampling techniques for the evaluation of treatment plans. Medical Physics, 20(1):151-161, 1993.

${ }^{9}$ G. J. Kutcher, C. Burman, L. Brewster, M. Goitein, and R. Mohan. Histogram reduction method for calculating complication probabilities for three-dimensional treatment planning evaluations. Int J Radiat Oncol Biol Phys, 21:137-146, May 1991.

${ }^{10} \mathrm{~K}$.H. Chadwick and H.P. Leenhouts. The molecular theory of $\mathrm{ra}-$ diation biology. Springer-Verlag, Berlin, Heidelberg, New York, 1981.

${ }^{11}$ W.H. Press, S.A. Teukolsky, W.T. Vetterling, and B.P. Flannery. Modeling of data. In Numerical Recipes: The art of scientific Computing. Cambridge University Press, Cambridge University Press, 1993.

${ }^{12}$ Amr Aref, Dale Thornton, Emad Youssef, Tony He, Samuel Tekyi-Mensah, Lori Denton, and Gary Ezzell. Dosimetric improvements following 3d planning of tangential breast irradiation. Int. J. Radiat. Oncol. Biol. Phys., 48:1569-1574, December 2000.

${ }^{13}$ P. M. Evans, V. N. Hansen, W. P. M. Mayles, W. Swindell, M. Torr, and J. R. Yarnold. Design of compensators for breast radiotherapy using electronic portal imaging. Radiotherapy and Oncology, 37:43-54, October 1995. 


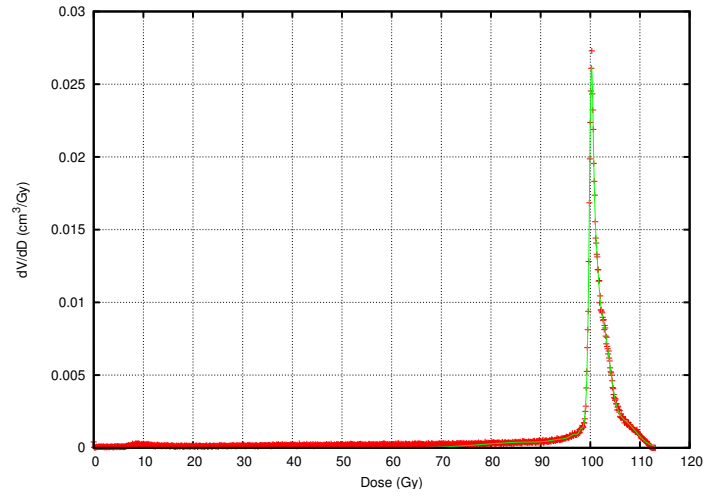

(a)Compensator — Target

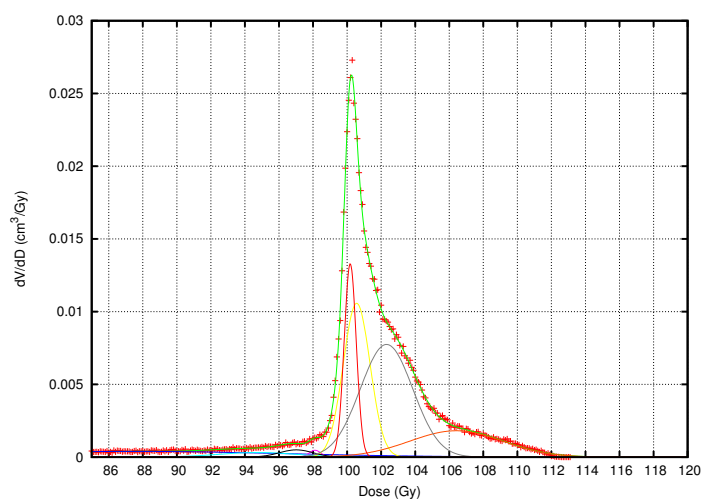

(c)Compensator — Target (zoom)

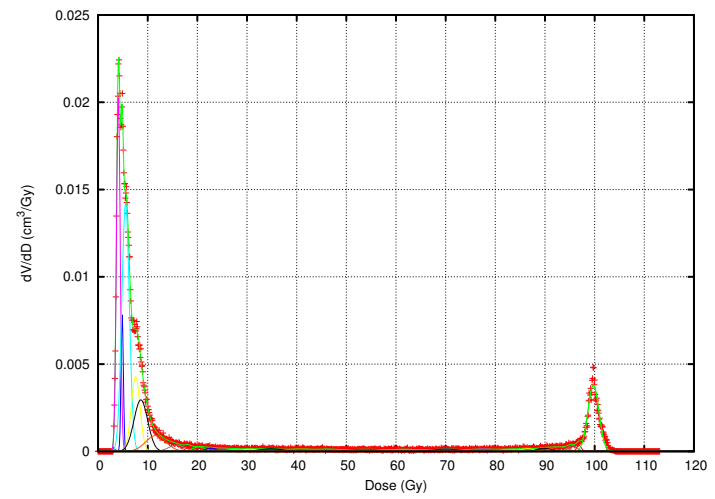

(e)Compensator — Ipsilateral breast

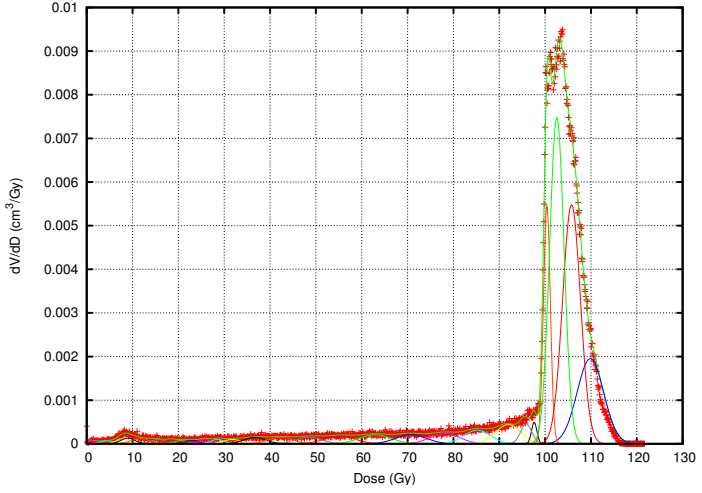

(b)Wedges - Target

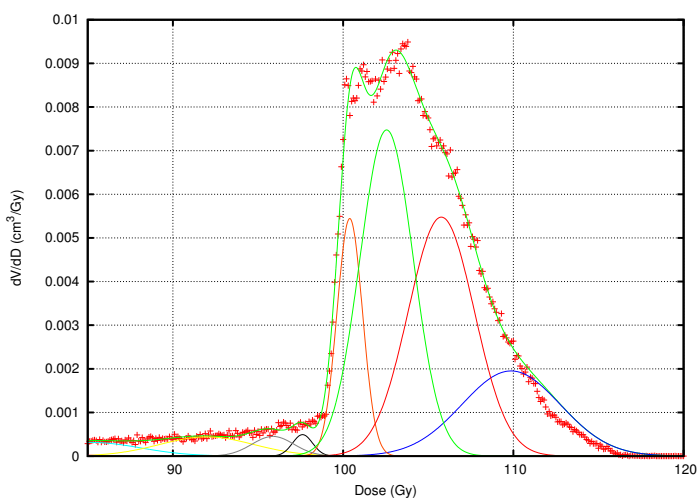

(d)Wedges - Target (zoom)

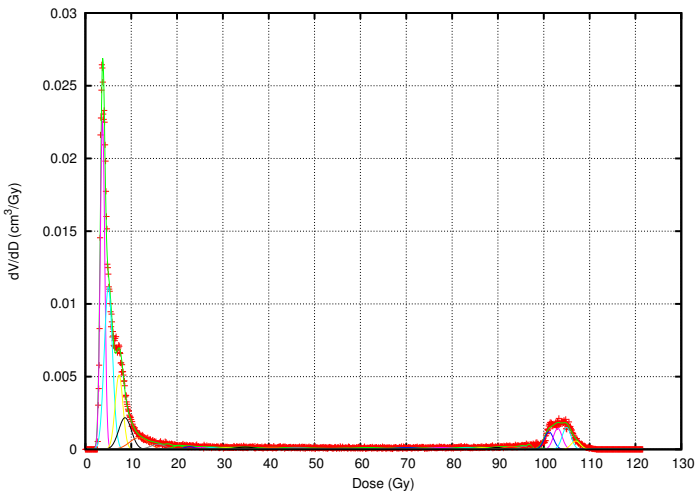

(f)Wedges - Ipsilateral breast

FIG. 3. Two different treatment approaches for breast carcinoma using two opposed tangential fields are shown. One using wedges, the other compensators. The dose distribution is normalized to a single point as being 100 Gy or $100 \%$. This implies that \% and Gy can be exchanged liberally. The original data is shown as a point plot, the fit as solid lines. The dDVH for the target, a zoomed plot of the target, and ipsilateral lung are shown. In the latter case it is clear that there are two separate regions. One hotspot and the rest of the lung receiving scatter from the treated volume. The compensator case is clearly more uniform which is also clearly expressed in the $(\sigma)_{\text {tot }}$ and $\left(x_{0}\right)_{\text {tot }}$ parameters. 


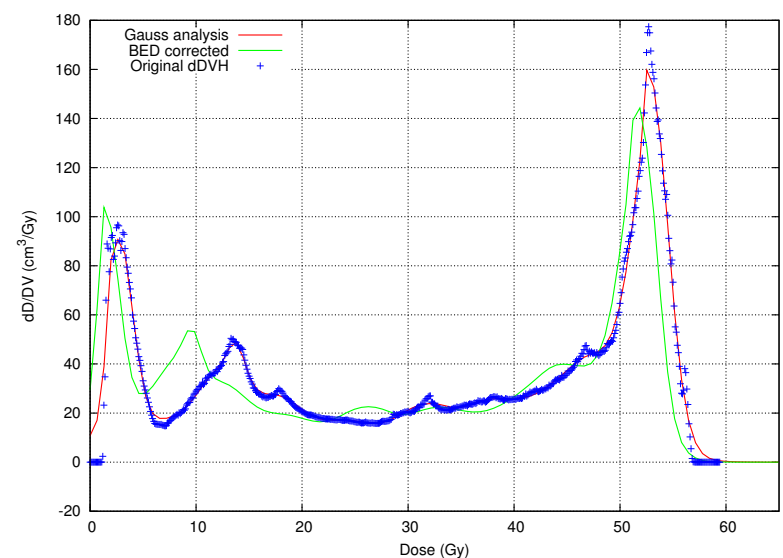

(a)Corrected DVH

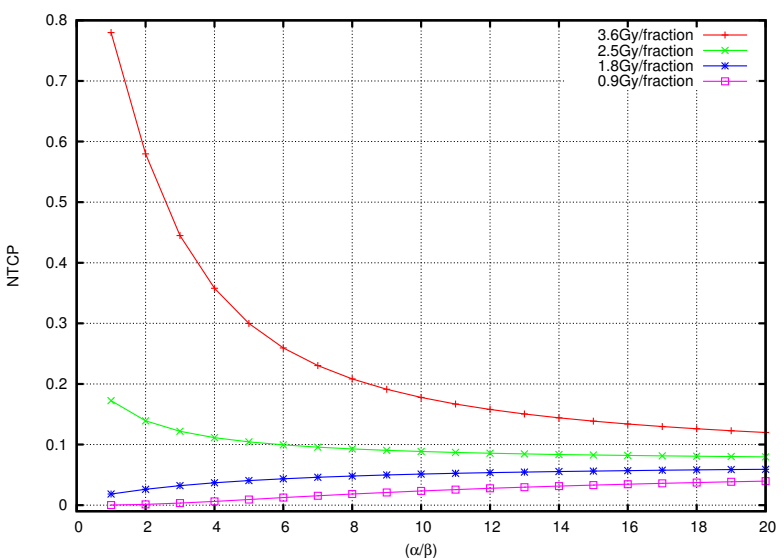

(b)Impact of varying $\frac{\alpha}{\beta}$

FIG. 4. The dDVH of the original data (point plot) normalized to the BED value of a 50.4 Gy delivered in fractions of 1.8 Gy is shown. Superimposed is the BED corrected dDVH. The curves in the left figure are adjusted using an $\alpha / \beta=10$. The total NTCP as calculated using the adjusted curves is dependent on the choice of $\alpha / \beta$ particularly in the hypo-fractionated regime with low $\alpha / \beta$-values $(1-3)$. This is illustrated in right figure.

${ }^{14}$ W. P. M. Mayles, J. R. Yarnold, and S. Webb. Improved dose homogeneity in the breast using tissue compensators. Radiotherapy and Oncology, 22:248-251, December 1991.

${ }^{15}$ C. J. McGinn, R. K. Ten Haken, W. D. Ensminger, S. Walker, S. Wang, and T. S. Lawrence. Treatment of intrahepatic cancers with radiation doses based on a normal tissue complication probability model. Journal Of Clinical Oncology: Official Journal Of
The American Society Of Clinical Oncology, 16:2246-2252, June 1998.

${ }^{16}$ Jackie Harney, Susan C. Short, Nihal Shah, Michael Joiner, and Michele I. Saunders. Low dose hyper-radiosensitivity in metastatic tumors. Int. J. Radiat. Oncol. Biol. Phys., 59:11901195, July 2004. 